Documentation et bibliothèques

\title{
La formation des bibliotechniciens hier, aujourd'hui et demain
}

\section{Françoise Jobin-Brûlé, Kathleen Mennie de Varennes et Léonard Nadeau}

Volume 19, numéro 2, juin 1973

\section{Bibliotechnique}

URI : https://id.erudit.org/iderudit/1055809ar

DOI : https://doi.org/10.7202/1055809ar

Aller au sommaire du numéro

\section{Éditeur(s)}

Association pour l'avancement des sciences et des techniques de la documentation (ASTED)

\section{ISSN}

0315-2340 (imprimé)

2291-8949 (numérique)

Découvrir la revue

\section{Citer cet article}

Jobin-Brûlé, F., de Varennes, K. M. \& Nadeau, L. (1973). La formation des bibliotechniciens hier, aujourd'hui et demain. Documentation et bibliothèques, 19(2), 51-56. https://doi.org/10.7202/1055809ar

\section{Résumé de l'article}

Les cours en bibliotechnique au Québec remontent au premier stage en bibliothéconomie de La Pocatière en juillet 1964. Le collège de Jonquière fut, en 1966, le premier à offrir des cours reconnus par un diplôme d'études collégiales. Trois cegep l'offrirent en 1966, deux en 1969, un en 1971, un en 1972. Deux autres collèges l'offriront, l'un en 1973 et l'autre en 1974. Deux comités du ministère de l'Education travaillent à l'élaboration des programmes et à l'étude du marché du travail. Au Canada anglophone, treize institutions offrent des cours de bibliotechnique; ces cours durent une année ou deux. A cause des difficultés d'emploi, un nouveau programme de Techniques de la documentation sera mis sur pied prochainement. Des normes pour l'enseignement de la bibliotechnique ont été proposées à la DIGEC à l'automne $1972 \ldots$
Tous droits réservés (C) Association pour l'avancement des sciences et des techniques de la documentation (ASTED), 1973
Ce document est protégé par la loi sur le droit d'auteur. L’utilisation des services d'Érudit (y compris la reproduction) est assujettie à sa politique d'utilisation que vous pouvez consulter en ligne.

https://apropos.erudit.org/fr/usagers/politique-dutilisation/ 


\section{La formation des bibliotechniciens hier, aujourd'hui et demain}

\author{
Françoise Jobin-Brûlé \\ Cegep Lionel-Groulx \\ Ste-Thérèse-de-Blainville \\ Coordonnateur provincial de bibliotechnique
}

avec la collaboration de:

Kathleen Mennie de Varennes

Ministère des terres et forêts

Québec

Agent de liaison de l'ACBLF auprès du Committee on the Traınıng of Library Technicians de la CLA Léonard Nadeau

Cegep de Rouyn-Noranda, Rouyn

Les cours en bibliotechnique au Québec remontent au premier stage en bibliothéconomie de La Pocatière en juillet 1964. Le collège de Jonquière fut, en 1966, le premier à offrir des cours reconnus par un diplôme d'études collégiales. Trois cegep l'offrirent en 1968, deux en 1969, un en 1971, un en 1972. Deux autres collegges l'offriront, I'un en 1973 et l'autre en 1974. Deux comités du ministère de l'Education travaillent à l'élaboration des programmes et à l'étude du marché du travail. $\mathrm{Au} \mathrm{Ca}$ nada anglophone, treize institutions offrent des cours de bibliotechnique; ces cours durent une année ou deux. A cause des difficultés d'emploi, un nouveau programme de Techniques de la documentation sera mis sur pied prochainement. Des normes pour l'enseignement de la bibliotechnique ont été proposées à la DIGEC à l'automne 1972. . .

\section{Les stages}

Au Québec, l'origine des cours en bibliotechnique remonte au premier stage en bibliothéconomie organisé au Collège Ste-Annede-la-Pocatière en juillet 1964. Lorsque l'université Laval annonce, en 1963, qu'elle discontinue ses cours d'été en bibliothéconomie, les étudiants déçus demandent à Raymond Boucher, directeur de la bibliothèque du collège de la Pocatière, de prende la relève. L'idée lui plaît et il commence alors à élaborer des plans.

Par ailleurs, à la Fédération des collèges classiques (FCC) la Commission des directeurs de bibliothèques et le Service des bibliothèques viennent d'être créés. La première équipe de direction de ces deux organismes se réunit. Elle étudie le projet de Raymond Boucher et décide de commencer des stages en bibliothéconomie à l'intention du personnel de soutien des bibliothèques de collège. La bibliothèque du collège de Ste-Anne-deLa-Pocatière organise les cours, publie un manuel tandis que la FCC patronne le projet. Les stages en bibliothéconomie de La Pocatière étaient nés. Au cours de 1963-1964, Raymond Boucher rédige le Manuel des tâches, recrute des professionnels et des techniciens d'expérience pour les assister, et plusieurs cours techniques comme les acquisitions, le catalogage et le classement de fiches, sont offerts. Les inscriptions atteignent soixante-cinq (65) pour le premier stage en juillet 1964 .

La théorie est réduite au minimum, l'accent étant placé sur le travail pratique exécuté sous la surveillance étroite de tuteurs. Les heures de travail sont très longues pour tous, aussi bien étudiants que tuteurs et professeurs. Mais les résultats sont encourageants: chacun, convaincu d'avoir beaucoup appris, veut revenir l'été suivant. D'autres cours sont offerts: les systèmes de classification Dewey et Library of Congress, les vedettes-matière, etc. Quelques anciens stagiaires deviennent tuteurs, puis professeurs. Le nombre d'étudiants augmente chaque année pour atteindre trois cent soixante (360) stagiaires en 1968. Des manuels et des cahiers d'exercices sont rédigés, expérimentés et publiés par la Société du Stage. De 1964 à l'été 1971, le contenu des cours de La Pocatière varie passablement d'une année à l'autre. En effet, les stagiaires sont des gens préoccupés par des problèmes concrets et il faut répondre à leurs besoins. Des diplômés d'université en bibliothéconomie participent à des sessions de perfectionnement, de sorte que les stages de la Pocatière sont connus depuis plusieurs années dans tout le Québec et même à l'extérieur. Ils sont une influence décisive sur le développement des bibliothèques au Québec. Un grand nombre de bibliothèques adoptent les méthodes enseignées à La Pocatière créant une certaine uniformité inconnue jusqu'alors.

\section{Les collèges}

En 1966, plus d'un an avant la création des cegep, le dynamique directeur des études du collège classique de Jonquière cherche à créer des cours techniques de niveau collégial. Le Rapport Parent paru depuis deux ans insiste sur le développement des cours tech- 
niques. Gérard Arguin rencontre Louise Gagnon qui avait participé aux stages de 1964 et de 1965 à La Pocatière; celle-ci accepte de relever le défi. Une trentaine d'étudiants s'inscrivent en septembre 1966 à l'institut de Technologie de Jonquière. Les cours se donnent cependant au collège classique. Le programme ressemble à celui de La Pocatière pour ce qui est de la bibliothéconomie mais, selon les recommandations du Rapport $\mathrm{Pa}$ rent, il comporte aussi des cours de culture générale.

A l'automne 1968, les cegep de RouynNoranda, de Trois-Rivières et de Maisonneuve à Montréal offrent le programme de bibliotechnique 390. L'année suivante, les cegep de Hull et Lionel-Groulx à Ste-Thérèse offrent aussi ce programme.

En 1971, le cegep François-Xavier-Garneau de Québec donne, à son tour, le programme 390. En 1972, le John Abbott College de Ste-Anne-de-Bellevue offre les premiers cours de bibliotechnique aux anglophones. On prévoit qu'à l'automne 1973, le Champlain Regional College de Lennoxville offrira aussi le programme ainsi que le cegep Montmorency à Laval en 1974. Le cegep Edouard-Monpetit offrit à l'éducation permanente des cours de bibliotechnique de 1967 à 1972.

Quand la Société du Stage en Bibliothéconomie de La Pocatière discontinue ses stages à l'été 1971, les dossiers des étudiants sont transférés au cegep François-Xavier-Garneau qui offre maintenant à son tour les cours d'été.

\section{La direction générale de l'enseignement collégial}

Deux comités sont formés par la DIGEC: la comité consultatif et le comité provincial de révision des programmes.

Le comité consultatif, créé en 1968, se composait de douze membres représentant divers groupements intéressés au milieu des bibliothèques ${ }^{1}$. Son mandat comportait l'étude des points suivants:

"relevé quantitatif et qualitatif de la profession afin de connaître les besoins actuels et futurs; définition des tâches de la profession et établissement d'un schème de programme correspondant aux différents niveaux d'activité; établissement de la liste d'équipement nécessaire à l'enseignement de la bibliothéconomie; aménagement des locaux utilisés à l'enseignement de cette spécialité; qualifications et recrutement du personnel enseignant; recyclage des individus actuellement sur le marché du travail; organisation de stages pratiques pour les étudiants; localisation géographique de la spécialité dans les cegep; tout autre moyen d'améliorer la formation professionnelle des spécialistes en bibliothéconomie; salaires et conditions de travail des bibliotechniciens".?

Le comité provincial de révision des programmes de bibliotechnique, créé à l'automne 1968, se composait de quatre responsables de l'option bibliotechnique, d'un représentant du comité consultatif et d'un représentant de la DIGEC. Le rôle de ce nouveau comité était d'adapter et d'améliorer les contenus de cours, d'étudier la méthodologie de l'enseignement et l'évaluation des étudiants.

En simplifiant beaucoup, on pourrait dire que le comité consultatif devait étudier le marché du travail et tracer un portrait idéal du bibliotechnicien, alors que le comité de révision avait pour fonction d'améliorer le programme de cours pour permettre d'atteindre cet idéal. Le comité consultatif relève directement du ministère de l'Education alors que le second relève du Service des Programmes de l'Enseignement Collégial.

Le mandat général de ces comités, version 1972, est le suivant:

"Le comité consultatif doit faire l'inventaire de la main-d'oeuvre qui dessert actuellement le secteur du monde du travail et en prévoir les fluctuations possibles durant les quelques années à venir; analyser les fonctions de travail du secteur donné en classifiant les opérations selon le degré de complexité des connaissances qu'elles exigent, adresser au ministère et au Service de l'Organisation de l'enseignement de la DIGEC ses recommandations touchant l'implantation de l'enseignement de telles(s) spécialité(s) dans certains CEGEP de la province, compte tenu des besoins en main-d'oeuvre décelés dans une

1. Les organismes représentés sont: l'Association canadienne des bibliothécaires de langue française, l'Association des bibliothécaires du Québec/Quebec Library Association, la Special Libraries Association (Montreal Chapter), I'Association des archivistes du Québec, l'Ecole de bibliothéconomie de l'université de Montréal, la School of Library Science de l'université McGill, le Service des bibliothèques d'enseignement du ministère de l'Education, le Service des bibliothèques publiques du ministère des Affaires culturelles, la Direction générale de l'enseignement collégial, la Fédération des collèges classiques remplacée par le Centre d'animation et de développement de la recherche en éducation (CADRE), le ministère du Travail. Les bibliothèques gouvernementales ont aussi un représentant.

2. Compte rendu de la première réunion tenue à Québec le 29 février 1968. 
étude antérieure; adresser au ministère et au Service des programmes de la DIGEC ses recommandations touchant les structures générales des programmes à dispenser dans les établissements, recommandations inspirées de l'étude des fonctions de travail et de leur évolution". 3

Le comité provincial de révision des programmes est

\begin{abstract}
"composé de professeurs spécialisés représentant chacune des institutions dispensant de telles spécialités, auxquels viennent s'adjoindre deux ou trois représentants du comité consultatif provincial de ce même secteur du monde du travail. Le mandat du comité provincial de révision des programmes consiste à habiller les structures générales de programmes recommandés par les membres du comité consultatif provincial, à les interpréter en unités d'enseignement, à les situer dans les cadres généraux des programmes du collégial; à prévoir les méthodologies propres à cet enseignement, à apporter aux programmes les modifications qu'ils jugent à propos de suggérer (. . .); à recommander l'adoption de certains manuels scolaires jugés particulièrement utiles à l'enseignement des diverses matières et à faire toute recommandation de nature pédagogique concernant l'application et l'orientation de tels programme". 4
\end{abstract}

Ce mandat précise que, par suite de la complémentarité de ces deux organismes, il tient,

\begin{abstract}
"pour assurer entre eux une osmose jugée indispensable, à ce que le président du comité provincial de révision des programmes ou le coordonateur puisse assister aux assemblées régulières du comité consultatif; de même, que deux ou trois délégués du comité consultatif puissent régulièrement siéger au comité provincial de révision des programmes". 5
\end{abstract}

\section{La formation au Québec}

A l'heure actuelle, au Québec, le programme de bibliotechnique se compose de onze (11) cours de bibliotechnique, de deux (2) cours de dactylographie, d'une session complète de stages dans trois types bibliothèques (d'enseignement, publiques et spécialiséé). Les futurs bibliotechniciens reçoivent aussi la formation de tout étudiant du niveau collégial: quatre (4) cours de philosophie, quatre (4) cours de français, quatre (4) cours

3. DIGEC, Mandat général, p. 1.

4. Ibid, p. 1

5. Ibid, p. 3. d'éducation physique et un certain nombre de cours obligatoires: histoire des sciences et des techniques, chefs-d'oeuvre de la littérature universelle, administration et relations humaines, initiation à la programmation, histoire de l'art et esthétique et quelques cours d'anglais. Ils doivent choisir quatre autres cours complémentaires. Avec cet éventail de cours, ils sont, en principe, familiarisés avec tous les rouages du travail de bibliothèque et possèdent une culture de base. Ils peuvent acquérir, cataloguer, classifier, prêter tout genre de documents; ils peuvent aussi classer les fiches, exécuter des recherches bibliographiques, aider à la référence, participer à des projets d'animation, assister le bibliothécaire au niveau des tâches administratives. Les premiers groupes d'étudiants se souviennent certes de l'anxiété qu'ils éprouvaient face à l'attitude du personnel des bibliothèques qui craignait que ces nouveaux venus ne prennent leur place. Les universitaires croyaient qu'on préférerait employer un bibliotechnicien qui serait payé moins cher, et le commis pensait qu'on le remplacerait par un bibliotechnicien diplômé. II ne semble pas que les personnes compétentes aient perdu leur place à cause des bibliotechniciens.

\section{La formation au Canada}

Ailleurs au Canada, les cours de bibliotechnique ont débuté en 1962 au Vancouver City College et au Red River Community College de Winnipeg. Vingt-trois (23) institutions post-secondaires offrent des cours de bibliotechnique dont neuf (9) en Ontario, une (1) au Manitoba, une (1) en Saskatchewan, deux (2) en Alberta, aucune dans les Maritimes ni à Terre-Neuve en plus des huit déjà mentionnées au Québec auxquelles il faut ajouter le collège Loyola. Tenant compte de la population et de la situation actuelle des bibliothèques au Québec, il est pour le moins surprenant de constater que nous possédons autant de cours de bibliotechnique que l'Ontario. Par ailleurs, il est visible qu'il existe un écart marqué entre les provinces de l'Ouest et les Maritimes, et il conviendrait qu'au moins un cours de cette catégorie soit disponible dans les provinces atlantiques le plus tôt possible. Dans le reste du pays, sauf pour ce qui est de Lakehead University à Thunder Bay et du Ryerson Polytechnical Institute à Toronto, les cours se donnent dans les Colleges of Applied Arts and Technology ou dans les Community Colleges. 
Au Québec, le cours dure trois ans. Ailleurs au Canada, il est de deux ans, à l'exception de la Saskatchewan Institute of Applied Arts and Sciences et du Red River Community College de Winnipeg qui offrent un cours d'un an.

Au Canada, comme au Québec, des cours de bibliotechnique sont aussi offerts par l'éducation permanente. Les personnes admises à ces cours doivent posséder un diplôme de 11e année et travailler dans une bibliothèque (cette condition n'implique pas nécessairement qu'elles possèdent une expérience pertinente). Ce n'est plus un secret pour personne que présentement ces cours causent un grave préjudice aux gradués des cours réguliers sur le marché du travail.

Les centres de main-d'oeuvre du Canada ont offert cette année divers cours de bibliotechnique aux collèges François-Xavier-Garneau, Lionel-Groulx et Trois-Rivières. De fortes pressions devraient être exercées auprès du ministère de l'Education pour réduire les programmes d'éducation permanente à un niveau raisonnable en tenant compte surtout des possibilités d'emploi. SeIon les dernières statistiques, plus de quatre cents (400) étudiants suivent ces cours au Québec. Dans le reste du pays, on n'atteint pas ce chiffre, en dépit de concentrations marquées à certains endroits, par exemple, l'Algonquin College d'Ottawa qui, à lui seul, compte quatre-vingt-dix (90) étudiants cette année.

Les étudiants québécois effectuent quinze (15) semaines de stages pratiques. Ailleurs, selon les normes de la Canadian Library Association, on n'exige que l'équivalent de dix (10) jours par an dans une bibliothèque locale. Le bibliotechnicien anglophone est limité aux bibliothèques locales, il est donc plus ou moins favorisé selon la situation géographique de son institution.

Les comités veillant à l'organisation et à la surveillance des cours sont en Québec, de niveau provincial, tandis que dans les autres provinces, c'est un Local Advisory Committee qui s'en occupe. Ces comités locaux font l'étude préliminaire du marché du travail de la région, jugent de l'opportunité ou non de la création d'un nouveau cours, voient à l'application des normes provinciales au niveau des cours et des enseignants et essaient d'estimer la valeur ou la rentabilité des cours une fois les diplômés lancés sur le marché du travail, servant en quelque sorte de lien entre le collège et l'employeur et de mécanisme d'ajustement des cours aux besoins.
Comparant le nombre de finissants en bibliotechnique du Canada et du Québec, au cours des trois (3) dernières années, on constate que le Québec, à lui seul, en compte à peu près la moitié. Au Québec, 1,243 étudiants incluant ceux de l'education permanente se sont inscrits à ce cours en 1972-1973.

Au Québec, le principal problème recontré par les bibliotechniciens est sans contredit leur trop grand nombre, amenant ainsi des difficultés particulières au niveau de leur emploi. Dans le reste du pays, par contre, le problème majeur est celui de la non-mobilité de cette main-d'oeuvre d'une province à l'autre à cause, en bonne partie, de la disparité des programmes.

Cette comparaison avec le reste du pays nous a amené l'an dernier à nous poser quelques questions. Le module Bibliotechnique du Collège Lionel-Groulx, composé d'étudiants, de professeurs, de bibliothécaires, de bibliotechniciens et d'animateurs socio-culturels, s'est demandé si le programme 390 répondrait encore longtemps aux besoins du marché du travail québécois limité aux bibliothèques. La question se posait d'autant plus que ce marché était quasi saturé à cause des restrictions budgétaires de nos bibliothèques. En effet, les directeurs de bibliothèques, bien que fort satisfaits de nos étudiants, et trouvant que ceux-ci étaient mieux formes que le personnel entraîné sur place, ne prévoyaient pas que leurs budgets augmenteraient suffisamment pour absorber tous les diplômés. Les plus beaux éloges nous ont été décernés par le personnel des écoles de bibliothéconomie quand ils ont reproché à nos cours d'être "trop avancés". Bien des étudiants et des professeurs de bibliotechnique ont cru, de leur côté, que les écoles de bibliothéconomie devraient exiger comme prérequis au moins certains cours de bibliotechnique. Le futur professionnel aurait alors, par exemple, une initiation au catalogage au collège et un cours savant à l'université. Ce qui, sans doute, servirait à rehausser le niveau de la profession au Québec.

\section{Nouvelles orientations}

La remise en question du programme par le module bibliotechnique de Lionel-Groulx aboutit donc au printemps 1972 à un Mémoire pour la restructuration des cours de Bibliotechnique, présenté au comité de révision des programmes et au comité consultatif de la DIGEC, ainsi qu'au comité de bibliotechnique de l'ACBLF. Ce rapport souleva quelques objections de la part de ces comités et fut longuement discuté. La DIGEC confia cette 
année, le mandat au comité de révision des programmes de travailler à l'élaboration d'un nouveau programme. Nous avons donc travaillé à la préparation d'un programme de TECHNIQUES DE LA DOCUMENTATION avec spécialisation en archives, en bibliothèque, en librairie et en technique de l'enseignement et de l'apprentissage.

Au cours de notre travail, nous avons réalisé que la tradition était assez puissante dans le monde de la bibliothéconomie. Nous avons néanmoins essayé de mettre sur pied notre "tronc commun" en TECHNIQUES DE LA DOCUMENTATION avec les voies de sortie possibles actuellement. Le comité de révision des programmes de bibliotechnique travaille, depuis 1968, à mettre sur pied le programme qui répond le mieux aux besoins du milieu. Devant le développement des dépôts d'archives dans les institutions d'enseignement et les municipalités, et aussi face à la nouvelle politique du livre et à loi des librairies agréées il faudra bien des techniciens qualifiés pour collaborer à la bonne marche de ces organismes. D'autre part, nous croyons que le rôle du collège Montmorency en tant que collège-bibliothèque expérimental n'est pas de doubler les options existantes mais d'offrir de nouvelles spécialités dans la ligne même des méthodes nouvelles d'enseignement qu'il préconise. Une des caractéristiques de la conception pédagogique de Montmorency, le nouveau rôle du professeur, - exige une différenciation des tâches de l'enseignant. Nous pourrions former ce que les Américains appellent les "teacher assistants" ou "learning assistants".

Le moment était donc venu d'élargir les horizons des étudiants et d'en faire des personnes polyvalentes. Notre comité, formé de sept (7) bibliothécaires, tous responsables des cours dans leur collège respectif, s'est donc adjoint, avec l'approbation de la DIGEC, François Beaudin, archiviste à l'université de

Montréal, et Raymond Carignan des Editions France-Québec, président de l'Association des libraires du Québec. Ces deux personnes nous ont aidés de leurs conseils judicieux, et le nouveau programme a été présenté récemment au ministère de l'Education. II devrait entrer en vigueur dans les cegep québécois en septembre 1974 et les cours de spécialisation devraient se donner en septembre 1976.

Ce programme de TECHNIQUES DE LA DOCUMENTATION compte quinze (15) cours pour le tronc commun, pour un total de trente $(30)$ crédits. ${ }^{6}$

6. Un (1) crédit reconnaît l'atteinte d'objectifs correspondant à quarante-cinq (45) heures d'activités d'apprentissage de la part de l'étudiant moyen; voir Coordination 1972-1973, p.1.
- Introduction à la documentation

- Ouvrages de référence

- Dactylographie I et II

- Introduction à l'organisation de la documentation

- Initiation aux principaux systèmes de classification, vedettes-matière, thesaurus

- Initiation à la classification Dewey

- Initiation à la classification de la Library of Congress

- Administration de bureau

- Classement de documents et de fiches

- Initiation à la comptabilité

- Relations humaines et relations de travail

- Initiation à la programmation

- Travail de recherche

- Initiation à l'audio-visuel
3 crédits

3 crédits

4 crédits

3 crédits

1 crédit

1 crédit

1 crédit

1 crédit

2 crédits

1 crédit

3 crédits

2 crédits

2 crédits

3 crédits
Les cours de la spécialisation bibliothèque seraient les suivants:

- Service de catalogage

- Service des acquisitions et des périodiques 2 crédits

- Service du prêt

- Service de la conservation

- Animation et publicité

- Classification Dewey

- Classification de la Library of Congress

- La bibliothèque dans son milieu

- Stage
3 crédits

1 crédit

1 crédit

2 crédits

2 crédits

2 crédits

2 crédits

15 crédits
Quant aux cours de spécialisation en archives et en librairie, ils seront étudiés en juin après présentation des programmes par l'Association des archivistes du Québec et l'Association des libraires du Québec. Cependant, nous savons qu'en archives les cours prépareront le technicien au classement, au transfert des documents, aux opérations d'appareils reproducteurs de documents et aux méthodes de conservation. Le techicien de la documentation spécialisé en archives pourra assumer les tâches dites techniques telles que: dépôt et versement de documents; triage selon les normes établies par l'archiviste; opération d'un centre de préarchivage, élaboration de certains instruments de recherche, classement de fiches, repérage du 
matériel à l'intention des administrateurs ou chercheurs, etc. . ${ }^{7}$

Les cours de la spécialisation en librairie présenteront le produit à vendre, diront comment et à qui le vendre. On y présentera le livre, l'édition, la mise en marché avec l'information, la publicité et la promotion du livre, l'organisation de la librairie: statut juridique et fiscal, le magasin avec son équipement et ses éléments d'ambiance, l'étude du marché, la gestion et la rentabilité, la publicité et l'art de vendre, les divers marchés et les clientèles du libraire: marché scolaire, universitaire, les bibliothèques publiques, le livre religieux et le livre professionnel. ${ }^{8}$

\section{Les normes}

Au printemps de 1972, le comité consultatif de bibliotechnique entreprit, suite à son mandat original de 1968 et à un travail de Léonard Nadeau en 1971, de rédiger des normes pour l'enseignement de la bibliotechnique au Québec.

Un sous-comité, formé de Raymond Boucher, de Jacques Paradis et de Françoise Jobin-Brûlé, a travaillé longuement à la rédaction de ces normes. Diverses annexes ont été faites par les collèges offrant le programme Bibliotechnique: Liste d'ouvrages de consultation; Liste d'ouvrages et des périodiques en bibliothéconomie; Liste des documents, de l'équipement et du matériel nécessaire pour les travaux de laboratoire.

Ces normes ont pour objectif d'aider le comité à évaluer périodiquement la qualité de l'enseignement de la bibliotechnique dans les cegep et de servir de guide pour les collèges dans l'organisation de leur département de bibliotechnique. Elles insistent sur les qualifications du personnel enseignant et du responsable de la spécialité, elles recommandent également qu'au moins un (1) bibliotechnicien travaille à plein temps au département et qu'un professeur soit dégagé de tout enseignement pour s'occuper des stages qui font partie du programme. Elles recommandent aussi que les collèges offrant le programme 390 recrutent un nombre suffisant d'étudiants pour justifier l'engagement des professeurs et l'équipement complet de laboratoires, et que ces collèges exigent des étudiants qu'ils aient une motivation sérieuse, une bonne connaissance du français et une connaissance suffisante de l'anglais.

\footnotetext{
7. François Beaudin, 390.00 Bibliotechnique. Spécialité. Techniques d'Archives, p. 1 et 2.

8. Document de l'Association des libraires du Québec,
} $2 \mathrm{p}$.
Les Normes pour l'enseignement de la bibliotechnique dans les cegep (programme 390) furent expédiées en novembre 1972 à la DIGEC, par Anna Rovira, présidente du comité consultatif. Fernand Gauthier, directeur du Service de la recherche de la DIGEC rencontra les membres du comité consultatif et du comité provincial de révision des programmes le 16 mars dernier, après nous avoir écrit le 14 février 1973, que "l'administration pour les collèges de normes aussi précises que celles que vous proposez, ne nous paraît pas possible étant donné les politiques financières, salariales et pédagogiques auxquelles elles sont soumises et les nombreuses contraintes matérielles, humaines et financières qui en résultent pour certaines collèges".

Les deux comités concernés ont donc conclu que leurs normes étaient renvoyées aux calendes grecques.

Jacques Paradis, président du comité de bibliotechnique de l'ACBLF et secrétaire du comité consultatif, a donc remis sa démission comme membre dudit comité. Nous cherchons le moyen de faire adopter nos normes par le ministère de l'Education, car il nous faut nous assurer que les cours donnés au Québec sont valables. Les cours actuels sont bons malgré certaines lacunes à certains endroits, et il faudra s'assurer que le cours de TECHNIQUES DE LA DOCUMENTATION visera l'excellence dès ses débuts.

D'ailleurs, les normes de 1972 sont facilement adaptables et transformables pour le cours de TECHNIQUES DE LA DOCUMENTATION et les comités concernés y travailleront dès que le nouveau programme aura été approuvé par la DIGEC.

Les professeurs de bibliotechnique, sont inquiets de la situation réservée à leurs étudiants lors de leur entrée sur le marché du travail. Ils formeront donc en juin une "Association des professeurs des "Techniques de la documentation" et défendront la cause des futurs techniciens en archives et en librairie, ainsi que celle des bibliotechniciense 